\title{
INTERACTIVE 3D VELOCITY ANALYSIS AND MODEL BUILDING FOR STACKING AND TIME MIGRATION
}

C.T. DALE, M. GRISCOM, J.B. HARTMAN and G. KISSINGER Chevron Overseas Petroleum Inc., 6001 Bollinger Canyon Road, San Ramon CA 94583, USA

An interactive method has been developed for carrying out seismic velocity analysis that results in a three dimensionally tied and consistent velocity field for either stacking or time migrating 3-D seismic data (also grids of 2-D data). This method uses interactive horizon-based velocity picking and statistical data fitting as the primary model building tools. The motivation for this development comes from difficulties we experience in picking seismic velocities from data which is either poor in its signal to noise ratio characteristics or is structurally complex and requires complicated velocities to image the subsurface.

This 3-D method for performing velocity analysis is, by choice, directly tied to the seismic data and interpreted time horizons. Figure 1 illustrates the major steps in the process. As input, this interactive method requires seismic data which has been processed into "cubes" of constant velocity DMO stacks or prestack migrations (Fowler, P., 1988). A fine enough velocity increment is chosen to prevent aliasing of either velocity or dip between adjacent constant velocity panels (typical velocity increments equal $50 \mathrm{~m} / \mathrm{s}$ ). An interactive "velocity cube" slicing and dicing program enables the interpreter to build a velocity function and output an optimum stack or migration for the 2-D analysis line location (Bishop, T. and R. Casserly, 1986). Velocity picking can be done by examining the constant velocity stacks, stacking power at selected mid-points, and stacking power along picked seismic horizons. A grid of lines from the survey area is analyzed in this way and then interpreted for geologically significant, consistent time structural horizons. Horizon velocity analyses along these key horizons are then extracted from the velocity cube data. Just as time horizons can be interactively interpreted, so can the corresponding velocity horizons. The result is a tied grid of time and velocity data (surfaces) that is objectively constrained by the seismic stack and migration images. Iterative review and refinement of the velocities takes place in section and map view. Simultaneously, the effects on the seismic imaging along control lines can be observed and reviewed through the velocity cube slicing process.

The velocity data volumes that result from this work are then used for velocity dependent data processing. The stacking velocity volume is typically left in a "raw" unsmoothed state to produce a stacked seismic volume. 3-D migration and/or depth conversion models are built using a geologically constrained fitting procedure which utilizes the mapped horizons as the velocity model framework. The large data volumes in a 3-D survey mean that there is a waiting period when large volume computational tasks are being run. Through careful project management it is possible to arrange for the data required for velocity analysis to be prepared prior to these more time 
consuming computer runs. Consequently the velocity work can be carried out in parallel to the standard signal processing flow. This approach separates 3-D seismic work into two areas of responsibility: the data processors are responsible for the management of the 3-D data volume and its processing and the interpretive personnel are responsible for the critical velocity analysis and model building steps. Consequently the geophysical model building becomes an integrated part of the data interpretation procedure. This methodology has been applied to a number of surveys offshore West Africa and will be illustrated with data examples from this area. Figure 2 shows a line from a 3-D survey, comparing the original 1987 processing with conventional velocity analysis and the new 1992 reprocessing with velocities picked using the interactive process described here (a nearby 2-D line is also shown).

\section{References:}

Bishop T. and R. Casserly, Geobyte V1, No1, 1986.

Fowler P., PhD Dissertation, Stanford University, 1988.

\section{INTERACTIVE 4-D VELOCITY ANALYSIS METHODOLOGY}

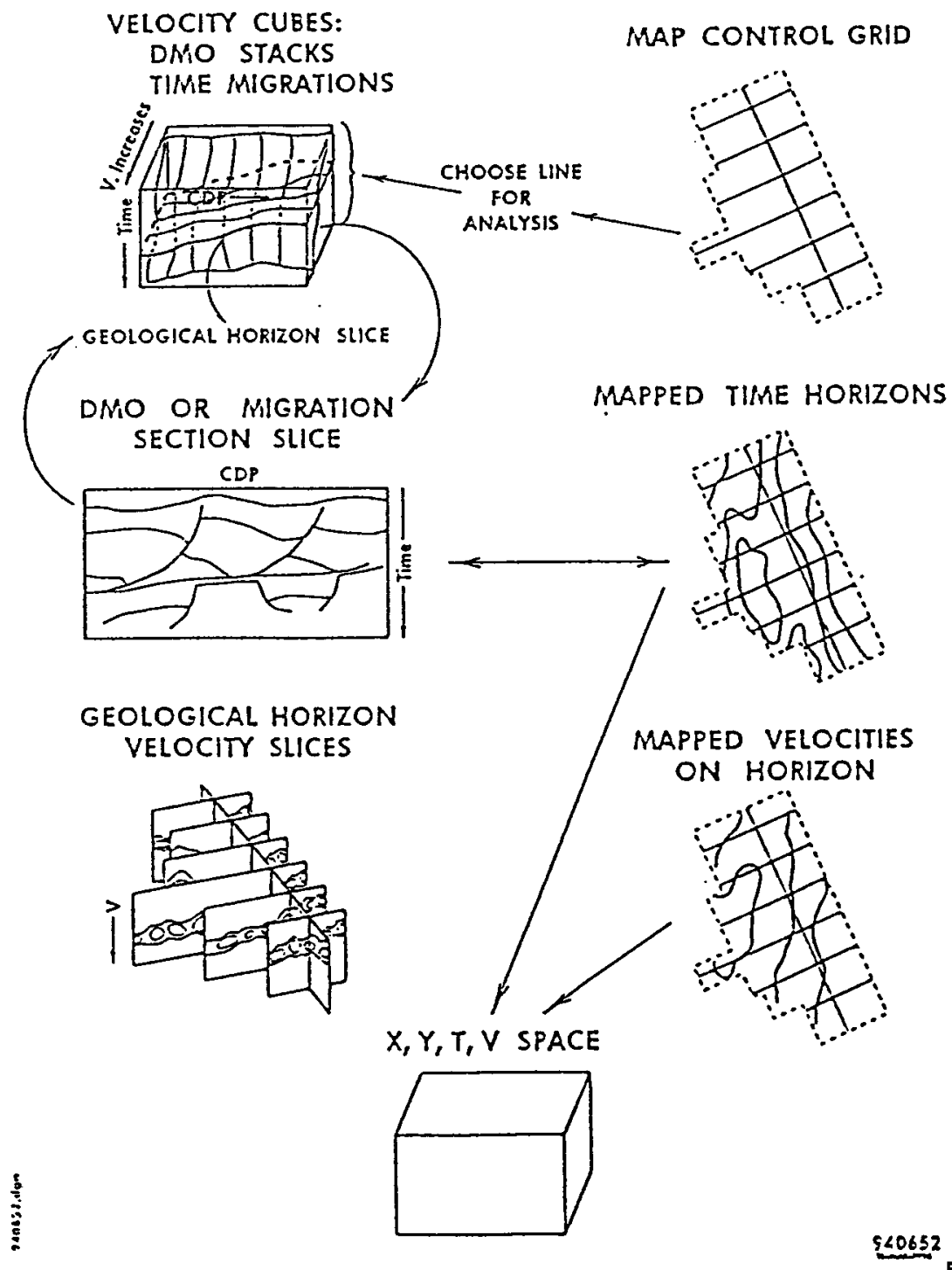


2-D DATA

…두

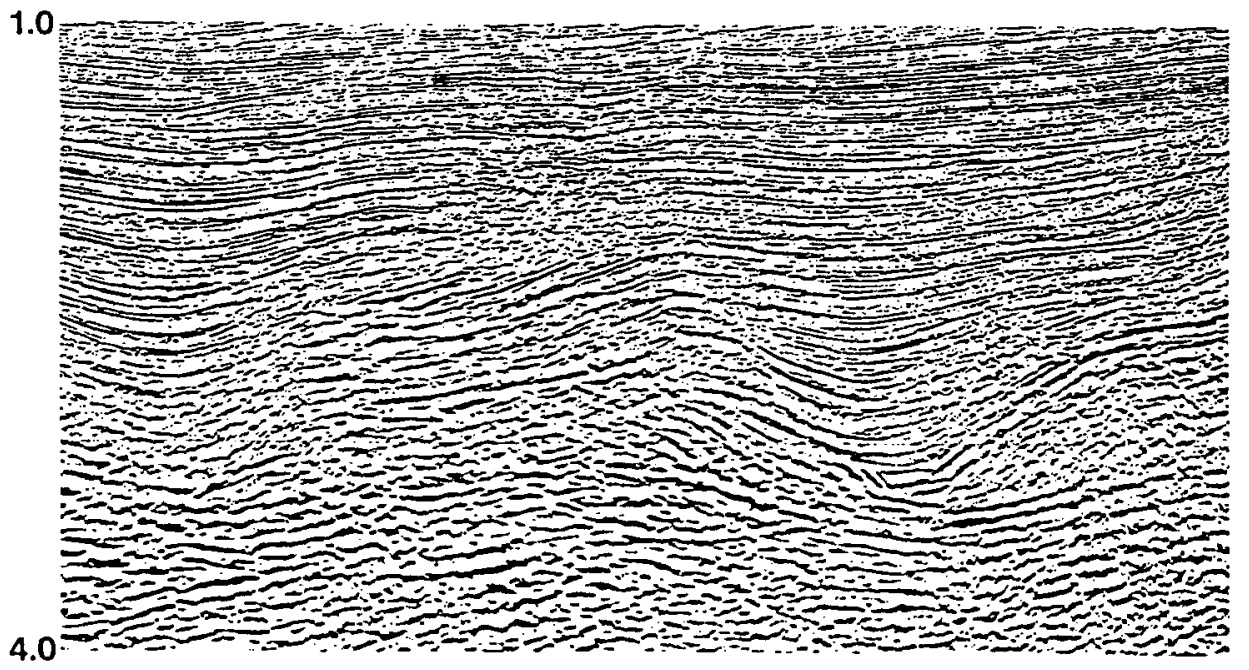

\section{3-D DATA, CONVENTIONAL VELOCITY ANALYSIS}

1.0

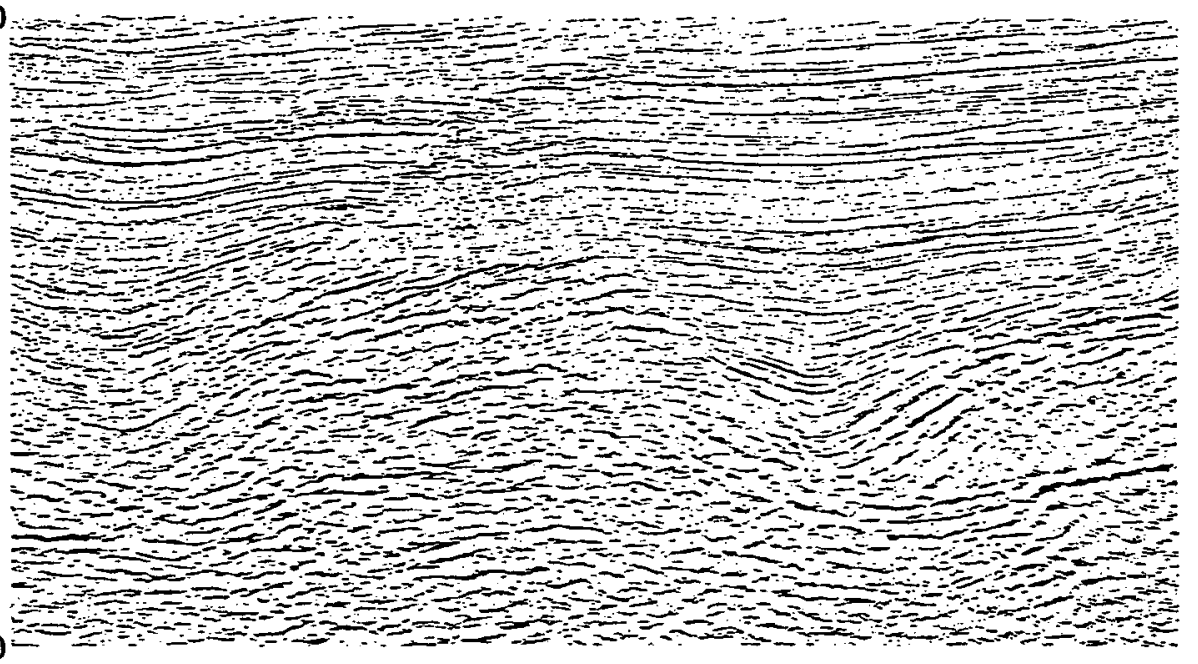

\section{3-D DATA, INTERACTIVE 3-D VELOCITY ANALYSIS}

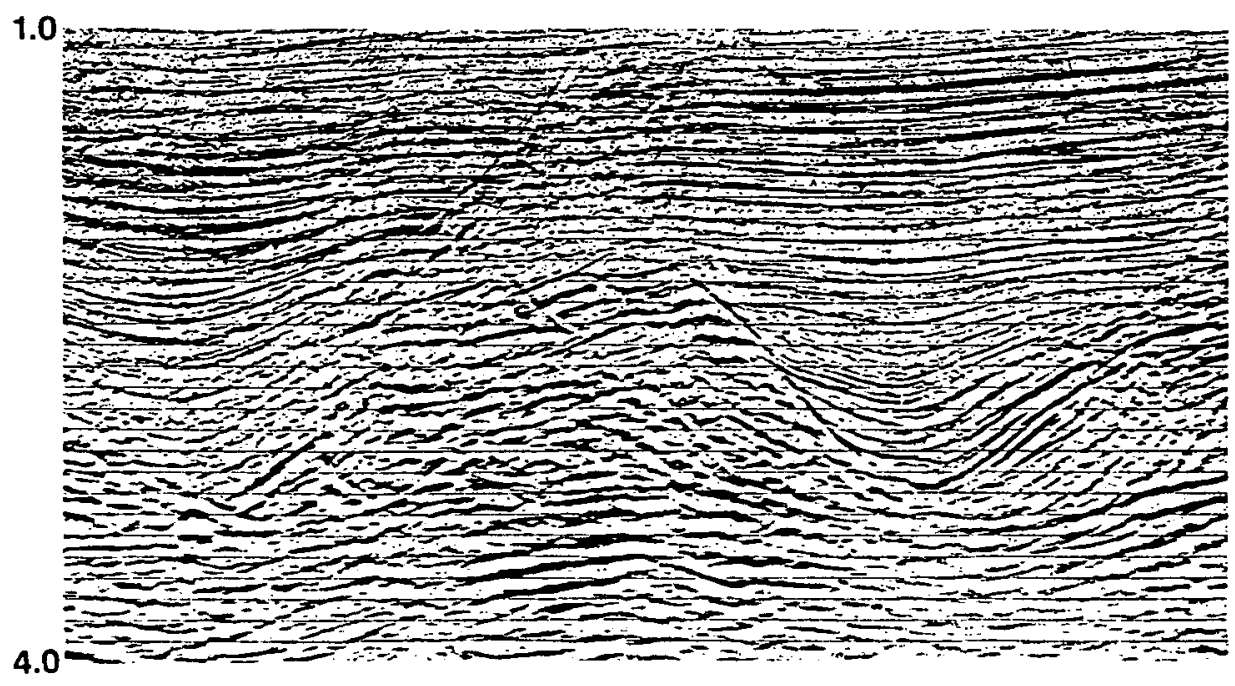



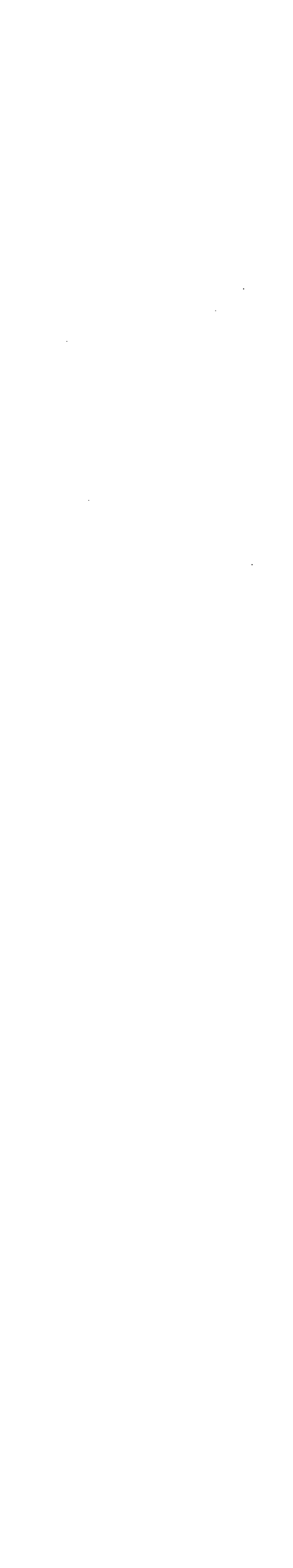